\title{
A Study to Identify the Risk Factors and their Relationship with Mucormycosis Severity in COVID Pneumonia
}

\author{
S. Lenus, M. Ramadevi, T. Muneeswar Reddy, C. Jaya Bhaskar, A. Bhavana
}

\section{ABSTRACT}

Background: Mucormycosis is an infection caused by a fungus that is present naturally in the environment. There has been an increased number of mucormycosis infections reported during COVID-19 and post COVID period. Dysregulated immune system due to COVID-19 and steroid use in the treatment which suppresses the immune system predisposes to this fungal infection. Patients with Diabetes Mellitus \& those who receive humidified oxygen during COVID-19 are found to be at higher risk of developing mucormycosis.

Methodology: 60 patients with mucormycosis admitted in the mucormycosis ward of SVRRGGH, Tirupathi, Andhra Pradesh, India in June 2021 are enrolled and retrospectively studied. Data were collected according to prestructured proforma about risk factors which include the previous history of Diabetes, CT severity score during COVID-19, and use of steroids and oxygen during the period of COVID-19.

Results: A total of 60 patients with mucormycosis were included in the study with a mean age of $49.25+/-10.29 .80 \%$ of the patients were males $\&$ $20 \%$ were females. $38.33 \%$ had mild COVID-19 (CTSS $0-7$ ), $45 \%$ had moderate disease (CTSS 8-17) and $16.66 \%$ had severe disease (CTSS 1825). $70 \%$ had stage $2,18.33 \%$ had stage 3 and $10 \%$ had stage 4 mucormycosis. Out of total patients, $78.33 \%$ were found to have a previous history of diabetes or newly detected diabetes. $60 \%$ of the patients had a history of humidified oxygen inhalation \& $58.33 \%$ of the patients had received steroids during COVID-19.

Conclusion: Male preponderance was observed in the study. Diabetes Mellitus, steroid use, humidified oxygen usage are the identifiable risk factors in mucormycosis associated with COVID-19. CT severity score is directly proportional to mucormycosis severity in the present study.

Keywords: COVID-19, CT severity score, diabetes mellitus, mucormycosis, steroid, oxygen.

\section{INTRODUCTION}

COVID-19 (coronavirus disease 2019) caused by severe acute respiratory syndrome coronavirus 2 (SARS-CoV-2) first reported in Wuhan, Hubei province of china progressed to a pandemic within a few months \& the second wave is continuing to affect the social \& economic life of people [1]. This unexpected havoc has been a medical terror worldwide for the past 2 years and has been given so many unanswered questions in front of the treating medical personnel. It has amazed the medical fraternity in terms of a wide variety of manifestations as well as complications and opportunistic infections. Mucormycosis is one of such opportunistic infections associated with COVID-19 [2].

Globally, the prevalence of mucormycosis was found to be
Published Online: February 1, 2022

ISSN: $2736-5476$

DOI: $10.24018 /$ ejclinicmed.2022.3.1.138

S. Lenus

Sri Venkateswara Medical College, Tirupati, Andhra Pradesh, India.

(e-mail: shilpalenus777@gmail.com) M. Ramadevi*

Sri Venkateswara Medical College, Tirupati, Andhra Pradesh, India.

(e-mail: ramadevimdgm@ ${ }^{@ m a i l . c o m) ~}$ T. Muneeswar Reddy

Sri Venkateswara Medical College, Tirupati, Andhra Pradesh, India.

(e-mail: drtmr2003@yahoo.co.in) C. Jaya Bhaskar

Sri Venkateswara Medical College, Tirupati, Andhra Pradesh, India.

(e-mail: jayabhaskar2002@gmail.com) A. Bhavana

Sri Venkateswara Medical College, Tirupati, Andhra Pradesh, India. (e-mail: bavu.asam@gmail.com)

*Corresponding Author 
Even though the fungal spores are normally found in the environment, our immune system is healthy enough to resist this infection in normal individuals [5]. Earlier, this infection was rare and was found to affect immunocompromised individuals. Uncontrolled diabetes, those with diabetic ketoacidosis, hematological and other malignancies, organ transplantation, prolonged neutropenia, immunosuppressive and corticosteroid therapy, iron overload or hemochromatosis, deferoxamine or desferrioxamine therapy, iv drug abusers, voriconazole therapy for transplant recipients, severe burns, AIDS, malnutrition, etc are risk factors for the development of this infection. Acidosis related to DKA and increased beta-hydroxybutyrate which increases the expression of the host \& fungal receptors result in fungal adherence $\&$ penetration into tissues thus predisposing to invasion [6].

It can affect the nose, sinuses, orbit, CNS, lungs, GIT, skin, joints, heart, kidney, and mediastinum (invasive type). But ROCM is the most common variety seen worldwide [6]. ROCM refers to the entire spectrum of mucormycosis ranging from limited nasal disease- stage 1(invasion of nasal cavity), sino-nasal disease-stage 2 (invasion from the nasal cavity to sinuses), rhino orbital disease-stage 3 (extension to orbits), and rhino-orbital-cerebral disease-stage 4 (further extension to the central nervous system) [6]. Patients present with complaints of unilateral retro-orbital headache, facial pain, numbness, fever, hyposmia, and nasal congestion which progresses to black discharge. Typical, black-colored necrotic eschars can be present over the nasal cavity, hard palate, or even over the face. Proptosis, ptosis, chemosis, and ophthalmoplegia indicate retro-orbital extension. Loss of vision can occur due to retinal artery thrombosis. Cranial nerves V and VII are the most common ones affected. Decreased level of consciousness suggests CNS involvement. In most cases, this infection is relentlessly progressive and results in death unless treatment with the combination of antifungal therapy and surgical debridement is initiated promptly [5].

Steroids have always been the cornerstone management in the treatment of COVID-19 to prevent cytokine storm. It has been speculated that dysregulated immune response associated with COVID-19 and steroids used in its treatment which suppresses the immune system predispose to this fungal infection [7]. Uncontrolled hyperglycemia and precipitation of DKA are often observed due to corticosteroid intake. Also, steroid use reduces the phagocytic activity of $\mathrm{WBC}$, causes impairment of bronchoalveolar macrophages migration, ingestion, and phagolysosome fusion making them more vulnerable to mucormycosis [3]. Hyperglycemia also causes glycosylation of transferrin and ferritin and reduces iron-binding allowing more free iron which is an ideal resource for mucormycosis. Increased levels of interleukin 6 and concomitant acidosis further increase free iron creating a favorable environment. It has been also postulated that the thrombotic pathophysiology which makes the COVID more dangerous than other viruses as the main mechanism which predisposes to mucormycosis also. Microthrombi occludes the capillaries and small arterioles leading to necrosis of tissues which creates an appropriate environment for fungal invasion. The characteristic property of mucorales for its aggressive nature is angioinvasion causing vasculitis and thrombosis of vessels resulting in infarction and necrosis of the involved area enhancing its ability to invade more tissue. Poor drug penetration to the dead tissue makes medical management less efficient [8].

The sudden rise in mucormycosis cases in COVID patients' needs much attention to assess the causative and predisposing factors in the scenario of COVID. It needs a special mention regarding the fact that this fungal infection has also been found to affect those patients even after the resolution of COVID symptoms. The sudden and drastic increase in the number of patients with mucormycosis during the pandemic poses a great challenge to the treating physicians. The mortality rate of mucormycosis in India ranges from $28-52 \%$ [9]. This warrants more study in this area to identify the risk factors associated with this infection. This study was undertaken primarily to identify the risk factors and their relationship with mucormycosis severity in COVID pneumonia patients. We also assessed the relationship between CT severity score and mucormycosis severity in COVID-19 patients as there are limited studies regarding it.

\section{MATERIALS AND METHODS}

This hospital-based retrospective observational study was conducted in S V R R Government General Hospital, Tirupati, Andhra Pradesh, India in June 2021. 60 patients admitted in mucormycosis ward during that period with a history of RT PCR or rapid antigen test (RAT) positive for SARS CoV 2 and age more than 18 years were taken as study subjects. Case records of the subjects who met the inclusion and exclusion criteria were collected from the medical records section and data was collected as per prestructured proforma. Patients were categorized into different stages of mucormycosis based upon anatomical involvement of structures as detected in CT or MRI Brain with paranasal sinus. The staging was done according to the proposed staging of rhino-orbital-cerebral-mucormycosis by [7] Patients were also categorized based on 25 point CT severity score during the period of COVID [10]. In statistical analysis, data were described as either categorical or continuous variables. Continuous variables were presented as mean with standard deviation and categorical variables as percentages. The Chi-square test and Pearson correlation coefficient were applied appropriately. Data were analyzed using SPSS Statistics 20.

\section{RESULTS AND ANALYSIS}

Out of the total 60 patients, $80 \%$ were males, and $20 \%$ were females. The age group ranged from 20 to 68 years in the study group. Most patients were between 51-60 years (41.67\%) both in males and females followed by $41-50$ years $(33.33 \%)$. Mean age of the study group was $49.25+/-10.29$ with males and females $49.68 \mathrm{yrs}$ and $47.17 \mathrm{yrs}$ respectively.

Out of the total 60 patients, $70 \%$ had stage 2 mucormycosis which involved nasal cavity and sinuses where $51.67 \%$ males and $18.33 \%$ females. Stage 3 mucormycosis was seen in $18.33 \%$ where all were males. $10 \%$ had invasive stage 4 mucormycosis involving CNS and only 1 patient had stage 1 mucormycosis limited to the nasal cavity. 


\section{GENDER DISTRIBUTION}

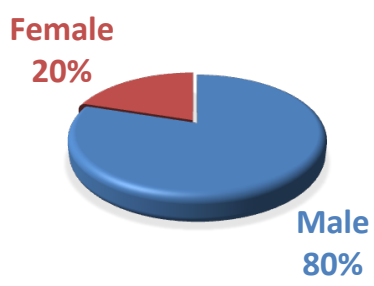

Fig. 1. Gender distribution.

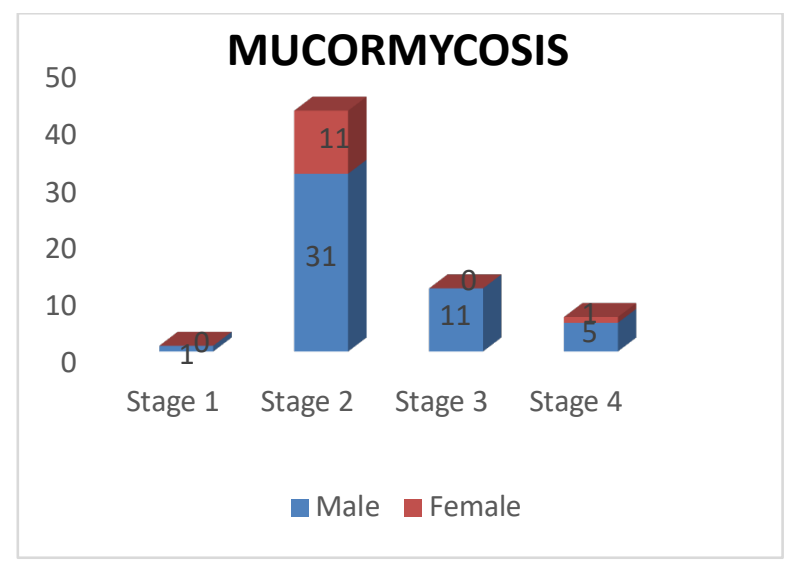

Fig. 2. Staging of Mucormycosis in relation to gender.

Steroid usage, oxygen inhalation, history of diabetes, and CT severity score during the period of COVID were studied. $78.33 \%$ were found to have a previous history of diabetes mellitus of which 3 patients had type 1 diabetes. In the diabetics, $63.33 \%$ were males and $15 \%$ were females. $60 \%$ of patients had a history of oxygen usage during the period of COVID of which $48.33 \%$ were males and $11.67 \%$ were females. $58.33 \%$ received steroids during COVID where $46.66 \%$ were males and $11,67 \%$ were females. The results in relation to diabetes history, oxygen use \& steroid use are shown in figure 3. Chi-square test was done to statistically analyze the correlation between the severity of mucormycosis with risk factors, but no significant correlation was found with diabetes $(p$ value $=0.061)$ oxygen use $(p$ value $=0.646)$ and steroid use ( $\mathrm{p}$ value $=0.642$ ).

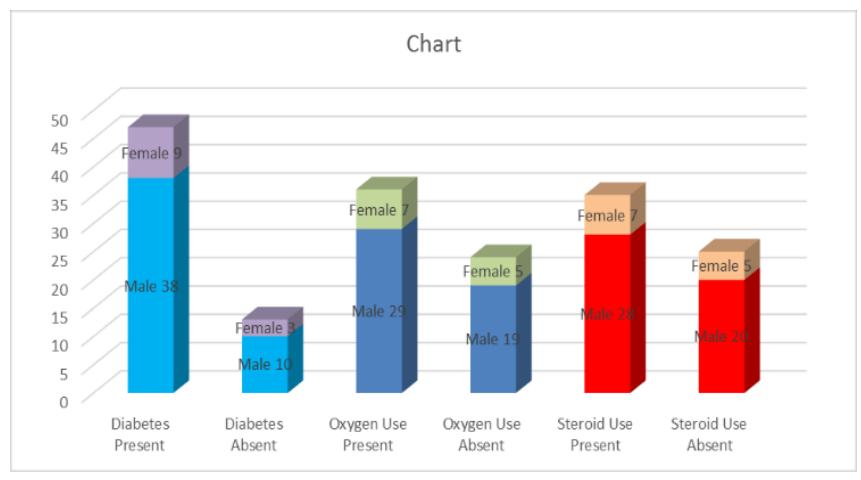

Fig.3. Risk factors in relation to gender.

In the study population, the mean CTSS was $9.35+/-0.739$. $38.33 \%$ had CT severity score $0-7$ (mild), $45 \%$ had CT severity score $8-17$ (moderate) and $16.66 \%$ had CT severity score 16-25 (severe). As CT severity score is a continuous variable, Pearson correlation was used for analysis which showed Pearson correlation $\mathrm{r}=0.275$, a small positive correlation was found between two variables. P-value was 0.033 ie; a significant correlation exists between CT severity. score and mucormycosis severity. The result is shown in Fig. 4.

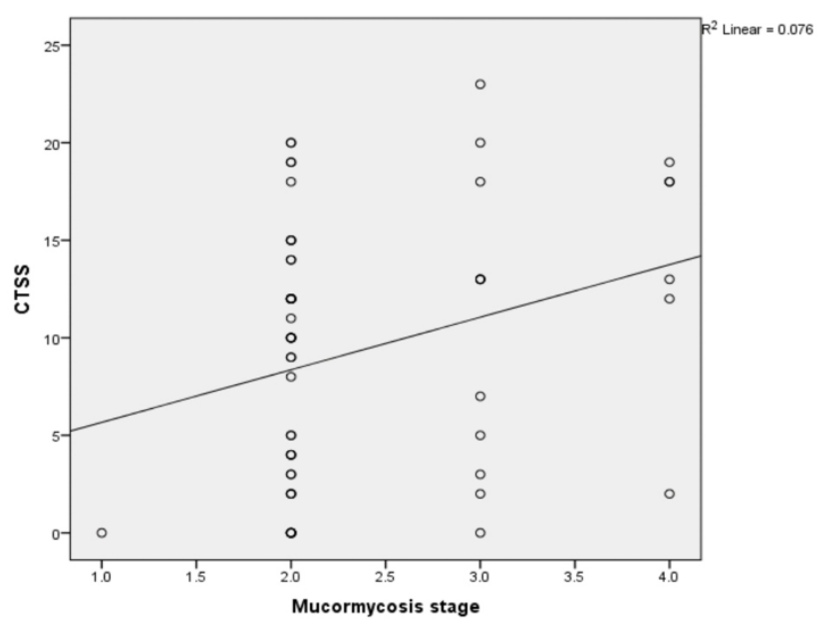

Fig. 4. Scatter diagram showing a positive correlation between mucormycosis stages and CT severity score.

\section{DISCUSSION}

Mucormycosis has emerged as one of the most important $\&$ life-threatening opportunistic infections associated with COVID-19. Several triggers may precipitate mucormycosis in COVID patients. In the prospective study by A Patel et al. including 465 patients done before the COVID era, predisposing factors for mucormycosis included diabetes mellitus $(73.5 \%)$, malignancy $(9 \%)$, and organ transplantation $(7.7 \%)$ [5].

In the present study, the majority of the study population were between $50-60$ yrs (41.67\%) followed by $41-50$ yrs $(33.33 \%)$ with a median age of 49.25 yrs. This finding was similar to the study by Chander et al where two-third of patients belong to age 31-60 yrs [11]. The study by A Ramaswami et al also had a median age of $44.5 \mathrm{yrs}$. $80 \%$ of our study subjects were males [12]. Male preponderance was also seen in other studies [1],[3],[8],[12],[13]. It has been hypothesized that estrogen might be protective in systemic fungal infections which could have led to a lower incidence in females [12].

$78.33 \%$ of our study subjects were diabetics, $60 \%$ had oxygen use and $58.33 \%$ had steroid use and $70 \%$ had stage 2 mucormycosis. Our results are consistent with the study by Awadesh Singh et al which included 101 patients in which male preponderance was present, $80 \%$ were diabetics, $76.3 \%$ had corticosteroid use and stage 2 was most common (88.9\%) followed by stage $3(56.7 \%)$ [3]. In another multicentric observational study by Mrittika sen et al. which included 2826 patients, similar results were found. ie, males were more affected (71\%), 78\% were diabetics, $87 \%$ had steroid use and $57 \%$ had oxygen need. But in their study, stage 3 was more common (72\%) followed by stage 2 [8]. Sharma et al. reported similar findings in their study of 23 patients where 21 had diabetes and all had received steroids. $43.47 \%$ had stage 3 mucormycosis in their study [14]. Y. Singh reported a case series of 14 patients with a median age of $49.5 \mathrm{yrs}$ 
similar to our study. Male predominance (78.5\%), Diabetes history in $61.5 \%$, and steroid use in $84.6 \%$ were observed.[13] The severity of COVID pneumonia was not correlated in any of these studies.

Our study found that mucormycosis severity was significantly correlated with COVID pneumonia as indicated by 25-point CT severity score. To the best of our knowledge, this study is one of the initial studies to correlate the severity of both mucormycosis and COVID pneumonia and there are limited studies regarding this correlation. In the case series by Sarkar et al, all 10 subjects were diabetics and had received steroids and male predominance was observed (80\%). All their patients had received oxygen with 9 patients requiring ventilator support indicating more severe COVID pneumonia [1]. COVID-19 often causes endothelialitis, endothelial damage, thrombosis, lymphopenia, and reduction in CD4+ and CD8+ T-cell level and thus predispose to secondary or opportunistic infections. This pathophysiology can be attributed to the result we found that mucormycosis severity increases as the severity of COVID pneumonia increases.

At the same time, it is to be noted that in our study, patients who are not diabetic $(21.67 \%)$, those who did not receive steroids $(41.67 \%)$ or oxygen $(40 \%)$ and was on home isolation have also developed this opportunistic infection which suggests other pathophysiology leading to it. Kumar et al. hypothesized that the excess of uncontrolled conventional precautions like repeated steam inhalation disrupts the nasal tract's beneficial microbiome and virome, thus suppressing local immunity and providing an opportunity to fungal infection [15]. Also during COVID-19, zinc has been used disproportionately. It has been proved that fungal cells must acquire zinc for their proper development during their life cycle and zinc deprivation by hosts is an important antifungal mechanism [16]. The study by Florencia Leonardelli et al. tried zinc chelators in vitro against mucorales and suggested that zinc depletion could be used as a treatment in mucormycosis [17]. Other factors like climate should also be taken into consideration because most infections are prevalent in the initial phases of hot and dry summer seasons when the temperature is high and relative humidity and precipitation are minimal [15].

\section{CONCLUSION}

Male preponderance is observed in the present study. The most common risk factor for COVID associated mucormycosis is diabetes mellitus. Oxygen inhalation \& steroids also increase the risk factor for this fungal infection. CTSS was significantly correlated with the severity of mucormycosis in the present study. Optimal management of diabetes and judicial use of steroids and oxygen should be recommended in COVID-19 patients.

\section{LIMITATIONS}

1. It is a retrospective study.

2. The duration between COVID-19 and the onset of mucormycosis was not assessed.
3. Diabetic control during the period of COVID-19 was not assessed.

4. Other risk factors, mode of oxygen delivery, type of steroids, duration of steroids were not considered.

\section{FUNDING}

This study did not receive any specific grant from funding agencies.

\section{CONFLICT OF INTEREST}

None to declare.

\section{REFERENCES}

[1] Sarkar S, Gokhale T, Choudhury SS, Deb AK. COVID-19 and orbital mucormycosis. Indian J Ophthalmol. 2021; 69: 1002-4.

[2] Alekesyev K, Didenko L, Chaudhry B. Rhinocerebral mucormycosis and COVID-19 pneumonia. J Med Cases. 2021; 12(3): 85-89.

[3] Singh AK, Singh R, Joshi SR, Misra A. Mucormycosis in COVID-19: A systematic review of cases reported worldwide and in India. Diabetes Metab Syndr. 2021; 15(4): 102146.

[4] Lackner N, Posch W, Lass-Flörl C. Microbiological and Molecular Diagnosis of Mucormycosis: From Old to New. Microorganisms. 2021; 9(7): 1518 .

[5] Patel A, Kaur H, Xess I. A multicentre observational study on the epidemiology, risk factors, management, and outcomes of mucormycosis in India. Clin Microbiol Infect. 2020; 26(7): 944.e9944.e15.

[6] Sugar AM. Mucormycosis. Clin Infect Dis. 1992; 14: S126e9.

[7] Honavar SG. Code Mucor: Guidelines for the Diagnosis, Staging, and Management of Rhino-Orbito-Cerebral Mucormycosis in the Setting of COVID-19. Indian J Ophthalmol. 2021; 69(6): 1361-1365.

[8] Sen M, Honavar SG, Bansal R, Sengupta S, Rao R, Kim U, et al. Epidemiology, clinical profile, management, and outcome of COVID19-associated rhino-orbital-cerebral mucormycosis in 2826 patients in India - Collaborative OPAI-IJO Study on Mucormycosis in COVID-19 (COSMIC), Report 1. Indian J Ophthalmol. 2021; 69(7): 1670-1692.

[9] Prakash H, Chakrabarti A. Epidemiology of Mucormycosis in India. Microorganisms. 2021; 9(3): 523.

[10] Saeed GA, Gaba W, Shah A, Al Helali AA, Raidullah E, Al Ali AB, et al. Correlation between Chest CT Severity Scores and the Clinical Parameters of Adult Patients with COVID-19 Pneumonia. Radiol Res Pract. 2021; 2021: 6697677.

[11] Chander J, Kaur M, Singla N, Punia RPS, Singhal SK, Attri AK, et al. Mucormycosis: battle with the deadly enemy over a five-year period in India. J Fungi 2018; 4: 46.

[12] Ramaswami A, Sahu AK, Kumar A, Suresh S, Nair A, Gupta D, et al. COVID-19-associated mucormycosis presenting to the Emergency Department-an observational study of 70 patients, QJM: An International Journal of Medicine. 2021.

[13] Singh Y, Ganesh V, Kumar S, Patel N, Aggarwala R, Soni KD, et al. (July 03, 2021) Coronavirus Disease-Associated Mucormycosis from a Tertiary Care Hospital in India: A Case Series. Cureus. 13(7): e16152.

[14] Sharma, S., Grover, M., Bhargava, S., Samdani, S., \& Kataria, T. Post coronavirus disease mucormycosis: A deadly addition to the pandemic spectrum. The Journal of Laryngology \& Otology. 2021; 135(5): 442447.

[15] Kumar M, Sarma DK, Shubham S, Kumawat M, Verma V, Singh B, et al. Mucormycosis in COVID-19 pandemic: Risk factors and linkages. Curr Res Microb Sci. 2021; 2: 100057.

[16] Staats CC, Kmetzsch L, Schrank A, Vainstein MH. Fungal zinc metabolism and its connections to virulence. Front Cell Infect Microbiol. 2013; 3: 65.

[17] Leonardelli F, Macedo D, Dudiuk C, Theill L, Cabeza MS, Gamarra S, et al. In Vitro Activity of Combinations of Zinc Chelators with Amphotericin B and Posaconazole against Six Mucorales Species. Antimicrob Agents Chemother. 2019; 63(5): e00266-19. 